enzymes. The latter includes the HECT domains, the RING finger domains, and the recently identified U-box domain ${ }^{12}$. Hakai belongs to the second group, as it has a RING finger domain highly related to that of the $\mathrm{Cbl}$ proto-oncoprotein. The relationship with $\mathrm{Cbl}$ may go beyond this domain and its function, as Hakai also exhibits a phosphotyrosine-binding domain and an extended proline-rich region that are highly related to those of Cbl. Although initial work indicated that the primary function of $\mathrm{Cbl}$ is to promote the downregulation of EGF receptors, a more complex picture has emerged from the realization that the internalization mechanism of a receptor can determine the repertoire of downstream signalling molecules it can activate ${ }^{13}$. In addition, Cbl itself is a substrate for tyrosine kinases and can function as an adaptor molecule, providing docking sites for the adapter protein CrkL and the p85 subunit of phosphatidylinositol-3-OH kinase ${ }^{14}$. Thus, $\mathrm{Cbl}$ may function as an adaptor molecule, as well as a regulator of receptor trafficking and signalling, by functioning as an E3 ubiquitin ligase.

Many lines of research have demonstrated that cadherins can function as signal-transducing molecules, in addition to their better-understood function in cell adhesion ${ }^{15}$. These observations raise the possibility that Hakai may also regulate the signalling properties of E-cadherin, together with its likely function in regulating the dynamic remodelling of E-cadherin-based adherens junctions, under both physiological and pathological conditions. The many therapeutic implications of understanding, and thus controlling, cadherin recycling and its regulation will certainly fuel the interest of basic and translational scientists alike in the race to evaluate these exciting possibilities, and to elucidate fully their underlying molecular mechanisms.

J. Silvio Gutkind is in the Oral and Pharyngeal Cancer Branch, National Institute of Dental and
Craniofacial Research and National Institutes of Health, Bethesda, Maryland 20892-4330, USA

Salvatore Pece is in the Department of

Experimental Oncology, European Institute of

Oncology, 20141 Milan, Italy

e-mail:sg39v@nih.gov

1. Kemler, R. Semin. Cell Biol. 3, 149-155 (1992).

2. Takeichi, M. Curr. Opin. Cell Biol. 7, 619-627 (1995).

3. Gumbiner, B. M. J. Cell Biol. 148, 399-404 (2000).

4. Fuijta et al. Nature Cell Biol. 4, 222-231 (2002).

5. Bonifacino, J. S. \& Weissman, A. M. Annu. Rev. Cell Dev. Biol. $14,19-57$ (1998).

6. Hicke, L. \& Riezman, H. Cell 84, 277-287 (1996).

7. Kamei, T. et al. Oncogene 18, 6776-6784 (1999).

8. Birchmeier, W. \& Behrens, J. Biochim. Biophys. Acta 1198, 11-26 (1994)

9. Batlle, E. et al. Nature Cell Biol. 2, 84-89 (2000).

10. Calautti, E. et al. J. Cell Biol. 156, 137-148 (2002).

11. Angst, B. D., Marcozzi, C. \& Magee, A. I. J. Cell Sci. 114, 625-626 (2001).

12. Patterson, C. Sci. STKE (cited 22 Jan 2002), http://stke.sciencemag.org/cgi/content/full/OC_sigtrans;2002/116/pe4 (2002).

13. Di Fiore, P. P. \& De Camilli, P. Cell 106, 1-4 (2001).

14. Thien, C. B. \& Langdon, W. Y. Nature Rev. Mol. Cell Biol. 2, 294-307 (2001).

15. Pece, S. \& Gutkind, J. S. J. Biol. Chem. 275, 41227-41233 (2000)

\title{
Ready and Abl
}

The actin cytoskeleton is a dynamic network that is affected by signals generated from either growth factors or extracellular matrix proteins. The reverse is also true, as rearrangements in the actin cytoskeleton can influence signal transduction. c-Abl, a nonreceptor tyrosine kinase, is at the interface of this crosstalk between growth-factor signalling, the extracellular matrix and cytoskeletal dynamics. c-Abl phosphorylates proteins involved in regulating actin dynamics; it also has an F-actin-binding domain (FABD). Because F-actin can inhibit c-Abl in vitro, it is highly likely that $\mathrm{c}-\mathrm{Abl}$ and $\mathrm{F}$-actin act together to regulate the actin cytoskeleton. Woodring et al. show in the latest edition of The Journal of Cell Biology (J. Cell Biol. 156, 879-892; 2002) that Factin and $\mathrm{c}-\mathrm{Abl}$ do indeed interact with one another to regulate the dynamics of cell morphology and motility.

Woodring et al. previously showed that purified c-Abl is inhibited by F-actin, an inhibition that can be relieved if the FABD in c-Abl is mutated. In suspended cells c-Abl colocalizes with F-actin, which correlates with a reduction in c-Abl kinase activity, but in adhered cells c-Abl dissociates from F-actin and remains dissociated at a steady state. This correlates with an increase in c-Abl kinase activity. Treating cells with latrunculin, which disrupts the structure of the actin cytoskeleton, prevents c-Abl and F-actin from associating, and increases the activity of c-Abl.

The authors also demonstrate that the formation of actin microspikes in fibroblasts that are spreading on fibronectin requires c-Abl, but not Src or the Rho family of GTPases (see figure; $\mathrm{c}-\mathrm{Abl}$ is shown in red and actin is shown in green). Although this activation of actin microspikes does not require the FABD, it does require c-Abl's kinase activity. This raises an interesting reciprocal interaction between $\mathrm{c}-\mathrm{Abl}$ and $\mathrm{F}$-actin. c-Abl can clearly activate actin microspike formation independently of the FABD, while at the same time F-actin can act to inhibit c-Abl

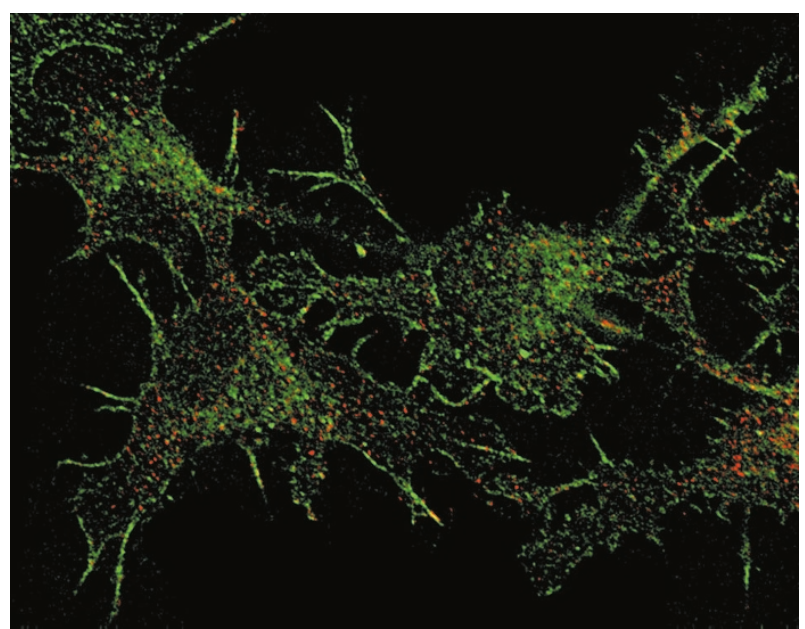

REPRODUCED WITH COPYRIGHT PERMISSION OF THE ROCKEFELLER UNIVERSITY PRESS (C)

activity via the FABD.

Clearly crosstalk between the actin cytoskeleton and c-Abl is more complicated than previously thought. Interactions between both c-Abl and F-actin might provide a feedback mechanism that allows cells to regulate actin cytoskeleton dynamics.

SARAH GREAVES 